\title{
The persistence of UCS intensity effects in acquired drive conditioning*
}

\author{
MELVIN L. GOLDSTEIN \\ Indiana University at Kokomo, Kokomo, Indiana 46901
}

\begin{abstract}
The persistence of conditioned fear was measured for varying intervals of time after conditioning in an acquired drive experiment. UCS intensity and number of CS-UCS pairings were varied at two levels during conditioning and the effects on a hurdle-jump test response were measured $24 \mathrm{~h}, 48 \mathrm{~h}$, and 7 days after conditioning. It was found that the effects of a high UCS intensity persisted for 7 days after conditioning, but that the effects a medium UCS intensity dissipated after $48 \mathrm{~h}$ after conditioning. The results were interpreted in terms of the relative contribution of UCS intensity to sHr and to D in the Spence-Hull system.
\end{abstract}

Miller and Lawrence (1950) have shown that differences in performance resulting from UCS intensity variations introduced during the classical conditioning phase of acquired drive conditioning persist to a second day of testing. They did not extend the testing period beyond $24 \mathrm{~h}$ after conditioning. McAllister and McAllister (1967) have also shown that UCS intensity variations during classical conditioning persist to a second day of testing. The effects of UCS intensity variations during the classical phase of acquired drive conditioning may, therefore, be relatively permanent and may contribute to $\mathrm{sHr}$, or learning, as well as to $\mathrm{D}$, or motivation (Spence, 1953, 1956). The drive construct (D), as applied to the analysis of the acquired drive experiment may, therefore, have relatively temporary (motivational) as well as permanent (associational) components (Hull, 1943).

The purpose of the present experiment was to determine the relative permanence of UCS intensity effects in acquired drive conditioning by extending the testing period beyond the usual $24 \mathrm{~h}$ after conditioning. It is assumed that if UCS intensity effects on performance persist beyond $24 \mathrm{~h}$, UCS intensity variations contribute primarily to sHr. If UCS intensity effects dissipate before $24 \mathrm{~h}$, then the independent variable probably contributes primarily to $D$. Contribution of UCS intensity to both $\mathrm{D}$ and sHr would be indicated by the finding of a temporary effect on performance, which lasts about $24 \mathrm{~h}$, and a more permanent residual emotional effect, which persists beyond $24 \mathrm{~h}$ after conditioning. The temporary portion of the effect should yield performance functions comparable to the stable functions obtained $24 \mathrm{~h}$ after conditioning (Goldstein, 1960), while the permanent portion of the effect should yield performance functions which show a decrement $24 \mathrm{~h}$ after classical conditioning

\footnotetext{
*Preparation of this report was supported in part by a supply grant and Summer Faculty Fellowships awarded by the Indiana University Office of Research and Advanced Studies (Grant 26-631-32). Charles Borneman, Jr., made the drawings, and Andy Elledge typed the manuscript. The writer wishes to thank his wife Daidee for her patience during the course of this work.
}

but still show some effect of UCS intensity on performance about $48 \mathrm{~h}$ after conditioning.

\section{METHOD}

\section{Experimental Design}

A 2 by 2 by 4 mixed factorial design, with repeated measurements of a hurdle-jump performance indicator, was used (Lindquist, 1953, pp. 281-284). Two levels of UCS intensity and two levels of number of CS-UCS pairings were used during the classical conditioning portion of acquired drive conditioning: 880 and $160 \mathrm{~V}$ ac, delivered to a grid floor through a fixed resistance of $250,000 \mathrm{ohms}$ (equivalent to 2.93 and $.53 \mathrm{~mA}$ ), and 1 and 20 CS-UCS pairings. The third dimension of the design represented the number of "instrumental" hurdle-jump test trials (four three-trial blocks) and was the same for all Ss. The hurdle-jump test period extended for 1,2, and 7 days beyond the classical conditioning session.

Ss were assigned at random to each of the four experimental groups. There were four $\mathrm{Ss}$ in each group.

\section{Subjects}

The Ss were 16 experimentally naive male albino rats, supplied by the Holtzman Co., Madison, Wisconsin. They were 140-150 days old at the start of the experiment and weighed from 416 to $513 \mathrm{~g}$.

\section{Apparatus}

The apparatus consisted of a black two-compartment conditioning chamber. One compartment contained a grid floor; the other compartment was a "safe" chamber with a wooden floor. The two compartments were separated by a guillotine-type door. The compound CS was a buzzer and light. The UCS was a 60 -cycle ac current conducted to each grid through a $250,000-\mathrm{ohm}$ series resistor. Further details of the apparatus have been described earlier (Goldstein, 1960).

\section{Procedure}

The procedure consisted of five experimental "days": on Day 1, Ss were adapted to the apparatus; on Day 2, the classical conditioning sessions were given; on Day 3 , the first hurdle-jump test session was given; on Days 4 and 5 , the second and third hurdle-jump test sessions were given. The first hurdle-jump test session was given $24 \mathrm{~h}$ after the classical conditioning session; the second hurdle-jump test session was given $48 \mathrm{~h}$ after the classical conditioning session; and the third hurdle-jump test session was given 7 days after the classical conditioning session. 
Adaptation to Apparatus (Day 1) $1 \mathrm{~h}$.

Each $\mathrm{S}$ was permitted to adapt to the grid compartment for

\section{Classical Conditioning (Day 2)}

CS and UCS were presented for 1 or 20 trials. The interval between CS and UCS onset was $4 \mathrm{sec}$, UCS duration was $1 \mathrm{sec}$, and CS and UCS were terminated simultaneously. Successive CS-UCS presentations were separated by 3-min intervals. All Ss remained in the conditioning compartment for the same length of time.

\section{Hurdle-Jump Test (Day 3)}

The effectiveness of the classical conditioning procedure was measured by requiring Ss to learn to jump across a hurdle from the grid floor into the wooden floor compartment when CS was turned on and the door separating the two compartments was opened. Ss were never shocked on this day and had never before been over the hurdle. Each $\mathrm{S}$ received 12 hurdle-jump acquisition trials with intertrial intervals of $8 \mathrm{~min}$. This session was conducted $24 \mathrm{~h}$ after the classical conditioning session.

\section{Second Hurdle-Jump Test (Day 4)}

The second test session was conducted $48 \mathrm{~h}$ after the classical conditioning session with the same procedure as the first test session.

\section{Third Hurdle-Jump Test (Day 5)}

The third test session was conducted 7 days after the classical conditioning session with the same procedure as the first test session, except that the intertrial intervals were $1 \mathrm{~min}$.

\section{RESULTS}

The hurdle-jump latency recorded on each test trial was transformed to a reciprocal. The mean of a block of three reciprocals was the score used in a series of analyses of variance.

The results of the analyses of variance indicated that the UCS intensity effect was significant beyond the .05 level of confidence on all 3 test days [Day $3, F(1,12)=$ 10.14; Day 4, F(1,12) = 7.43; Day 5, $F(1,12)=15.58]$.

Performance of the high UCS intensity, 20 CS-UCS pairings group was most responsible for the persistent UCS intensity effect (Fig. 1).

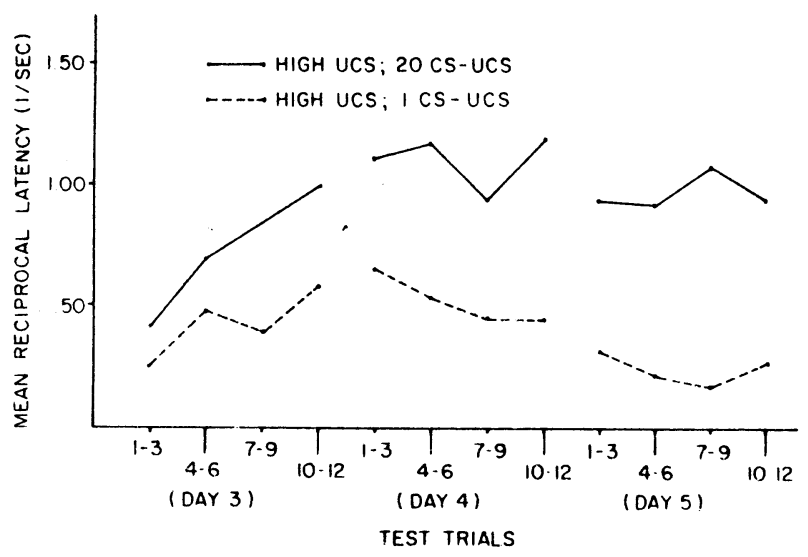

Fig. 1. Performance functions for the high UCS intensity groups on Days 3, 4, and 5 .
The performance level of the high UCS intensity, 20 CS-UCS pairings group remained stable from Days 3 to $4, F(1,3)=.50$. There was no significant change in the performance of this group from Day 4 to Day $5, F(1,3)$ $=1.44$.

The effect of the medium UCS intensity persisted only to the second day of testing (Day 4) and showed a decrement on the third day of testing (Day 5) (Fig. 2).

The decrement in performance of the medium UCS intensity, 20 CS-UCS pairings group from Day 4 to Day 5 was significant beyond the .01 level of confidence, $F(1,3)=103.33$. There was no significant change in performance from Day 3 to Day $4, F(1,3)=$ .67. The decrement in performance of the medium UCS intensity, 1 CS-UCS pairing group from Day 4 to Day 5 was just short of significant at the .05 level of confidence, $F(1,3)=6.00$. There was no significant change in performance of this group from Day 3 to Day 4, $F(1,3)=.10$. Edwards's $(1960$, p. 226) $F$ test for trend was used for the day to day comparison.

\section{DISCUSSION}

The major findings of this experiment were that a high intensity UCS used during classical conditioning produced a conditioned fear response which persisted for 7 days after the original conditioning session. A medium UCS intensity produced a less stable conditioned fear response which persisted for only $48 \mathrm{~h}$ after the original conditioning session.

The general findings of the present experiment tend to support the hypothesis that UCS intensity variations in acquired drive conditioning may contribute to both $\mathrm{sHr}$ and $\mathrm{D}$. A relatively permanent effect on performance was found at the high UCS intensity level, and a temporary effect on performance, which dissipated after $48 \mathrm{~h}$ after conditioning, was found at the medium UCS intensity level. UCS intensity, therefore, probably contributes to both $\mathrm{sHr}$ and $\mathrm{D}$, depending upon whether a high or medium UCS intensity is used during conditioning.

The findings of the present experiment corroborate the findings of Miller and Lawrence (1950). The performance functions for the second day of testing for the high and medium UCS intensity, 20 CS-UCS pairings groups are comparable to Miller and Lawrence's (1950) results for the second day of

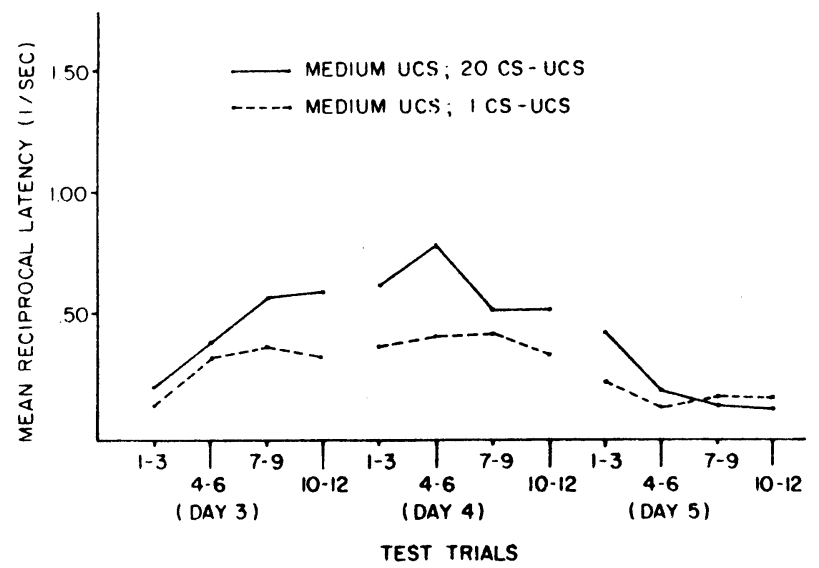

Fig. 2. Performance functions for the medium UCS intensity groups on Days 3, 4, and 5. 
testing. In both experiments, performance was maintained at a high level during the second test day. In the present experiment, however, performance showed a decrement 7 days after conditioning for the medium UCS intensity groups, but showed no such decrement for the high UCS intensity groups.

McAllister and McAllister (1968) have shown that there is a decrement in performance following a $47-\mathrm{h}$ interval between conditioning and test sessions. The UCS intensity used in their experiment $(.83 \mathrm{~mA})$ was comparable in magnitude to the medium UCS intensity $(.53 \mathrm{~mA})$ of the present experiment. The finding of a performance decrement 7 days after conditioning in the present experiment, with the medium UCS intensity, extends the findings of McAllister and McAllister (1968) to a longer time period after conditioning.

Further experiments are needed to explore the temporal course of the persistence of the conditioned fear response over longer time intervals such as a month after classical fear conditioning and to determine the nature of the cues responsible for the persistence of conditioned fears after conditioning with the high UCS intensity. It is quite likely that, after conditioning with the high UCS intensity, conditioned fear will persist for a longer period of time than measured in the present study. It is probable that if the test situation were made discriminably different from the conditioning situation, the conditioned fear response would extinguish rapidly.

\section{REFERENCES}

Edwards, A. L. Experimental design in psychological research. New York: Holt, Rinehart \& Winston, 1960.

Goldstein, M. L. Acquired drive strength as a joint function of shock intensity and number of acquisition trials. Journal of Experimental Psychology, 1960, 60, 349-358.

Hull, C. L. Principles of behavior. New York: A ppleton-Century-Crofts, 1943.

Lindquist, E. F. Design and analysis of experiments in psychology and education. Boston: Houghton Mifflin, 1953.

McAllister, D. E., \& McAllister, W. R. Forgetting of acquired fear. Journal of Comparative \& Physiological Psychology, $1968,65,352-355$.

McAllister, W. R., \& McAllister, D. E. Drive and reward in aversive conditioning. American Journal of Psychology, 1967, LXXX, 377-383.

Miller, N. E., \& Lawrence, D. H. Studies of fear as an acquirable drive: III. Effect of strength of electric shock as a primary drive and of number of trials with the primary drive on the strength of fear. Cited in S. S. Stevens (Ed.), Handbook of experimental psychology. New York: Wiley, 1951.

Spence, K. W. Learning and performance in eyelid conditioning as a function of the intensity of the UCS. Journal of Experimental Psychology, 1953, 45, 57-63.

Spence, K. W. Behavior theory and conditioning. New Haven, Conn: Yale University Press, 1956.

(Received for publication June 7, 1974.)

\title{
Constancy scaling theory and the Mueller-Lyer illusion: More disconfirming evidence
}

\author{
RICHARD GRIGGS*† \\ Indiana University, Bloomington, Indiana 47401
}

\begin{abstract}
Length and width judgments were made for modified Mueller-Lyer figures (the center shaft of each of the two figures was replaced by a rectangle). The rectangle with outgoing fins was judged longer and wider than a control rectangle of equal size with no fins, but longer and narrower than the rectangle with ingoing fins. The rectangle with ingoing fins was judged shorter and wider than the control rectangle. These results did not support Gregory's inappropriate constancy scaling explanation of the Mueller-Lyer illusion.
\end{abstract}

Gregory's inappropriate constancy scaling explanation has been responsible for much of the recent interest in the Mueller-Lyer illusion (Gregory, 1963, 1966, 1970). Gregory proposes that the Mueller-Lyer figures are perceived as flat projections of three-dimensional

*This research was supported by United States Public Health Service Grant PHS MH-16817 to Frank Restle. The author is grateful to Jerry Forshee for programming assistance and to Frank Restle (who sponsors the paper and takes full editorial responsibility) and Conrad Mueller for helpful comments on earlier drafts of this paper.

tThe research reported was done while the author was a graduate student at Indiana University; he is currently at the University of Florida. Requests for reprints should be sent to Richard A. Griggs, Department of Psychology, University of Florida, Gainesville, Florida 32611. displays, e.g., the figure with the outgoing fins (arrow feather figure) is a projection of an inner corner of a room and the figure with the ingoing fins (the arrowhead figure) is the projection of an outside corner of a building. Perspective cues provided by the fins in each figure cause the shaft of the arrow feather to be judged as farther away than the fins, and the shaft of the arrowhead figure to be judged closer than the fins. These cues inappropriately trigger the constancy scaling mechanism responsible for size constancy. This results in the expansion of the shaft in the arrow feather figure and the shrinkage of the shaft in the arrowhead figure producing the illusion. 